\title{
Perforin Is Detrimental to Controlliny C. muridarum Replication In Vitro, but Not In Vivo
}

\author{
Raymond M. Johnson ${ }^{1 *}$, Micah S. Kerr ${ }^{1}$, James E. Slaven ${ }^{2}$
}

1 Department of Medicine, Indiana University School of Medicine, Indianapolis, Indiana, United States of America, 2 Department of Biostatistics, Indiana University School of Medicine, Indianapolis, Indiana, United States of America

\begin{abstract}
CD4 T cells are critical for clearing experimental Chlamydia muridarum genital tract infections. Two independent in vitro CD4 $\mathrm{T}$ cell mechanisms have been identified for terminating Chlamydia replication in epithelial cells. One mechanism, requiring IFN- $\gamma$ and $\mathrm{T}$ cell-epithelial cell contact, terminates infection by triggering epithelial production of nitric oxide to chlamydiacidal levels; the second is dependent on T cell degranulation. We recently demonstrated that there are two independent in vivo clearance mechanisms singly sufficient for clearing genital tract infections within six weeks; one dependent on iNOS, the other on Plac8. Redundant genital tract clearance mechanisms bring into question negative results in single-gene knockout mice. Two groups have shown that perforin-knockout mice were not compromised in their ability to clear $C$. muridarum genital tract infections. Because cell lysis would be detrimental to epithelial nitric oxide production we hypothesized that perforin was not critical for iNOS-dependent clearance, but posited that perforin could play a role in Plac8-dependent clearance. We tested whether the Plac8-dependent clearance was perforin-dependent by pharmacologically inhibiting iNOS in perforin-knockout mice. In vitro we found that perforin was detrimental to iNOS-dependent CD4 T cell termination of Chlamydia replication in epithelial cells. In vivo, unexpectedly, clearance in perforin knockout mice was delayed to the end of week 7 regardless of iNOS status. The discordant in vitro/in vivo results suggest that the perforin's contribution to bacterial clearance in vivo is not though enhancing CD4 T cell termination of Chlamydia replication in epithelial cells, but likely via a mechanism independent of $\mathrm{T}$ cell-epithelial cell interactions.
\end{abstract}

Citation: Johnson RM, Kerr MS, Slaven JE (2013) Perforin Is Detrimental to Controllin $\gamma$ C. muridarum Replication In Vitro, but Not In Vivo. PLoS ONE 8(5): e63340. doi:10.1371/journal.pone.0063340

Editor: Kyle Ramsey, Midwestern University, United States of America

Received January 10, 2013; Accepted March 30, 2013; Published May 14, 2013

Copyright: (c) 2013 Johnson et al. This is an open-access article distributed under the terms of the Creative Commons Attribution License, which permits unrestricted use, distribution, and reproduction in any medium, provided the original author and source are credited.

Funding: This research was funded by an NIH grant R01Al070514. The funders had no role in study design, data collection and analysis, decision to publish, or preparation of the manuscript.

Competing Interests: Raymond Johnson and Indiana University School of Medicine have a patent PENDING related to this research titled GENETICALLY MODIFIED MICE FOR MODELING CHLAMYDIA TRACHOMATIS PATHOLOGY AND ASSISTING IN VACCINE DEVELOPMENT, 61/361,110. Raymond Johnson and the Indiana University Research and Technology Corporation are partial owners of a virtual biotechnology company called Zeta Immuno. At this time Zeta Immuno does not have intellectual property rights related to any of the information or technology present in this manuscript. The other authors have no conflicts of interest related to this manuscript. This does not alter the authors' adherence to all the PLOS ONE policies on sharing data and materials.

*E-mail: raymjohn@iupui.edu

\section{Introduction}

Chlamydia trachomatis genital tract infections have been, and continue to be, the most common bacterial sexually transmitted infections in Western societies [1]. Antibiotic-based public health measures have not decreased the incidence of infection and may be partially counterproductive, reducing Chlamydia immunopathology but also herd immunity [2-4]. Based on this epidemiologic reality many public health officials and sexually transmitted disease healthcare providers believe that a Chlamydia vaccine is the only intervention likely to reduce the incidence of infections and their associated monetary and personal costs.

Critical to a vaccine effort is defining the relevant host defense mechanism for protective immunity; i.e. the immunologic goal of vaccination. This is especially important for Chlamydia vaccines as early attempts with crude whole Chlamydia vaccines were associated with poor protection and excess immunopathology in humans and experimental primate models [5]. Investigations into Chlamydia genital tract protective immunity in the C. muridarum model have shown that CD4 $\mathrm{T}$ cells are critical to bacterial clearance [6], while CD8 $\mathrm{T}$ cells have been associated with immunopathology $[7,8]$.
Identification of an effector cell type does not equate a mechanism of protective immunity. The first identified mechanism for CD4 T cell-mediated termination of Chlamydia replication in epithelial cells was described in 1996. In vitro Chlamydia-specific Th1 cells terminated replication by inducing epithelial nitric oxide production to chlamydiacidal levels through a combination of IFN- $\gamma$ and $\mathrm{T}$ cell-epithelial cell contact $[9,10]$. However in vivo studies were discordant as mice deficient in inducible nitric oxide synthetase (iNOS) resolved C. muridarum genital tract infections $[11,12]$; suggesting that nitric oxide was either irrelevant to bacterial clearance or redundant in the setting of a second independent clearance mechanism.

We recently showed that clearance of $C$. muridarum from the genital tracts of C57BL/6 mice during 8 weeks following infection was dependent on having either a functional iNOS or Placo mechanism. Mice singly deficient nitric oxide production (treated with $i N O S$ inhibitor N-monomethyl-L-arginine, MLA) or deficient in Placo (genetic knockout) cleared C. muridarum genital tract infections at $\sim 6$ weeks compared to $\sim 4$ weeks for wild type mice. Dual deficient mice, genetically-deficient in Plac 8 and pharmacologically-deficient in $i N O S$ activity (MLA treated) were effectively 
unable to clear a genital tract infection over 8 weeks with 11 of 12 mice still shedding an average of $1300 \mathrm{IFU} /$ mouse on day 56 . However, both $i$ NOS-dependent (nitric oxide) and Plac8-dependent mechanisms were required for sterilizing immunity as viable $C$. muridarum could be recovered from genital tracts of Plac 8 knockout mice that had previously cleared a primary infection by delayed treatment with MLA [13]. This result mirrored that of Ramsey et al who showed that viable $C$. muridarum could be recovered from iNOS-knockout but not wild type mice that had previously cleared a genital tract infection when they were treated with the lymphocyte toxin cyclophosphamide [14].

Two previous studies have shown that perforin knockout mice were not compromised in their ability to clear $C$. muridarum genital tract infections. One study showed a trend toward less oviduct pathology in perforin knockout mice [15], the other a statistically significant reduction [8]. Because perforin knockout mice have an intact iNOS-clearance mechanism, it is possible that a role for perforin in Plac8-dependent clearance was masked by the $i N O S$ dependent clearance mechanism. With similar reasoning, we hypothesized that if perforin was necessary for the Plac8dependent mechanism, then a perforin knockout mouse treated with MLA would not clear a C. muridarum infection over 8 weeks.

Unexpectedly we found that perforin knockout mice were compromised in their ability to clear C. muridarum genital tract infections, and that the role of perforin in clearance is not likely through enhancing CD4 $\mathrm{T}$ cell termination of Chlamydia replication by either of the two known in vitro mechanisms dependent on iNOS [10] and T cell degranulation [16].

\section{Materials and Methods}

\section{Mice}

4-5 week old female C57BL/6J and C57BL/6-Prf1 ${ }^{\mathrm{tm} 1 \mathrm{Sdz} / \mathrm{J}}$ mice were purchased from The Jackson Laboratories (Bar Harbor, MA). All mice were housed in Indiana University School of Medicine specific-pathogen-free facilities (SPF). All animal experiments were approved by the AAALAC-accredited Indiana University School of Medicine Animal Care and Use Committee performed in accordance with the United States Animal Welfare Act and the National Research Council's Guide for the Care and Use of Laboratory Animals. Mice were lightly anesthetized with isoflurane for infections and vaginal swabs to minimize stress and discomfort.

\section{Genital Tract Infections}

One week prior to infection mice were treated with $2.5 \mu \mathrm{g}$ of medroxyprogesterone delivered subcutaneously (Depo-Provera, Pfizer Pharmaceuticals, New York, NY). Lightly anesthetized mice were infected vaginally with $5 \times 10^{4}$ inclusion forming units (IFU) of C. muridarum biovar Nigg in $10 \mu \mathrm{l}$ of SPG buffer. Mice were swabbed Tue-Thu to monitor the clearance of $C$. muridarum from the genital tract. Mice had access to water ad libitum, without or with $50 \mu \mathrm{M}$ N-monomethyl-L-arginine (EMD4biosciences; Billerica, MA). Water was supplemented as needed and exchanged entirely for fresh solutions twice a week for the duration of the experiment as in the previous publication [13]. Power analysis based on our previous Plac8 MLA publication [13] and a previous perforin knockout mouse study [15] showed that experimental groups of 5 mice had $97-99 \%$ likelihood to detect significant differences $(p$ value $<0.05)$ between groups if the assessment was done in the day 40-56 window post infection.

\section{Pathology Scoring}

A simple scoring system was utilized to assess macroscopic genital tract pathology [13]. Hydrosalpinx was scored as 0 , 1, or 2 reflecting no, unilateral, or bilateral hydrosalpinx respectively. Mice have a bi-fed uterus (2 uterine horns) that was similarly scored 0,1 , or 2 for hydro-uterus. The maximum pathology score for an individual mouse was 4 .

\section{Media, T cell Expansion, Epithelial Cells, and Bacteria}

$\mathrm{T}$ cell expansion cultures were grown in RPMI 1640 (Sigma) supplemented with $10 \%$ characterized fetal bovine serum (HyClone), $2 \mathrm{mM}$ L-alanyl-L-glutamine (Glutamax I; Gibco/ Invitrogen), $25 \mu \mathrm{g} / \mathrm{ml}$ gentamicin (Sigma), and $5 \times 10^{-5} \mathrm{M} 2$ mercaptoethanol (Sigma); referred to as RPMI CM. Immune splenocytes harvested from mice were plated at $12.5 \times 10^{6}$ cells per well in tissue culture treated 12 well plates, in RPMI CM containing murine recombinant IL- $1 \alpha$ (2 $\eta \mathrm{g} / \mathrm{ml})$, IL-6 (2 $\eta \mathrm{g} / \mathrm{ml})$, IL-7 (3 $\eta \mathrm{g} / \mathrm{ml})$, IL-15 (4 $\eta \mathrm{g} / \mathrm{ml})$, human recombinant IL-2 (100 units $/ \mathrm{ml}), 20 \% \quad 2^{\circ}$ MLC, and $10 \mu \mathrm{g}$ of UV-inactivated $C$. muridarum $(\sim 2.5$ IFU equivalents per splenocyte) as previously described [17]. Experiments were performed after the second passage/expansion. Polyclonal $\mathrm{T}$ cell populations were frozen at passages 3 and 4. C57epi.1 epithelial cells were cultured as previously described [17]. Mycoplasma-free Chlamydia muridarum (Nigg), previously known as C. trachomatis strain mouse pneumonitis (MoPn) (Nigg) was grown in McCoy cells as previously described [18].

\section{Cytokine ELISAs}

$3 \times 10^{6}$ immune splenocytes were mock-activated (SPG buffer) or active with $7.5 \times 10^{6}$ IFU UV-inactivated C. muridarum $(1200 \mathrm{~J} \times$ 2 in a Spectrolinker XL-1000 UV crosslinker) in 48 well tissue culture plates in $0.7 \mathrm{ml}$ of RPMI CM. Culture supernatant was harvested at $72 \mathrm{~h}$ and relative levels of IFN- $\gamma$, TNF- $\alpha$, IL-13, and IL-10 in culture supernatants determined by ELISA using capture and biotinylated monoclonal antibody pairs with recombinant murine standards according to the manufacturer's protocols. IFN$\gamma$ ELISA: XMG1.2 (Pierce-Endogen; Rockford, IL). TNF- $\alpha$ ELISA: TN3-19.12/C1150-14; IL-10 ELISA: JES5-2A5/SXC-1 (BD Biosciences; San Jose, CA). IL-13 ELISA: Ebio13a/ Ebio1316H (Ebioscience; San Diego, CA). Detection was accomplished with Streptavidin-HRP (BD Biosciences) and TMB substrate (Sigma Chemical Co., St. Louis, MO).

\section{Flow Cytometry}

Arbitrarily selected polyclonal T cell populations \#5 (wild type) and \#15 (perforin-deficient) were stained for $20 \mathrm{~min}$ on ice in RPMI CM with: PE-coupled 53-6.7 (CD8 $\alpha$ ) (BD Biosciences), FITC-coupled GK1.5 (CD4), FITC-coupled eBR2a (control antibody), PE-coupled LO-DNP-11 (control antibody) (Ebioscience; San Diego, CA). Cells were fixed with $1 \%$ paraformaldehyde after staining and analyzed on a FACSCalibur cytometer (BD Biosciences).

\section{Epithelial Cell Infections/Chlamydia Replication/Titration/ Microscopy}

C57epi.1 cell monolayers in 48 well plates were untreated or treated with IFN- $\gamma(10 \eta \mathrm{g} / \mathrm{ml})$ for $10 \mathrm{~h}$ prior to infection. Wells were infected with 3 IFU per cell. After addition of $C$. muridarum the plates were spun at $300 \times \mathrm{g}$ for $30 \mathrm{~min} .4 \mathrm{~h}$ post infection the inoculums were removed and CD4 $\mathrm{T}$ cell clones were added in RPMI CM. 32 h post infection, wells were scraped, harvested with an equal volume of sucrose-phosphate-glutamate acid buffer (SPG 

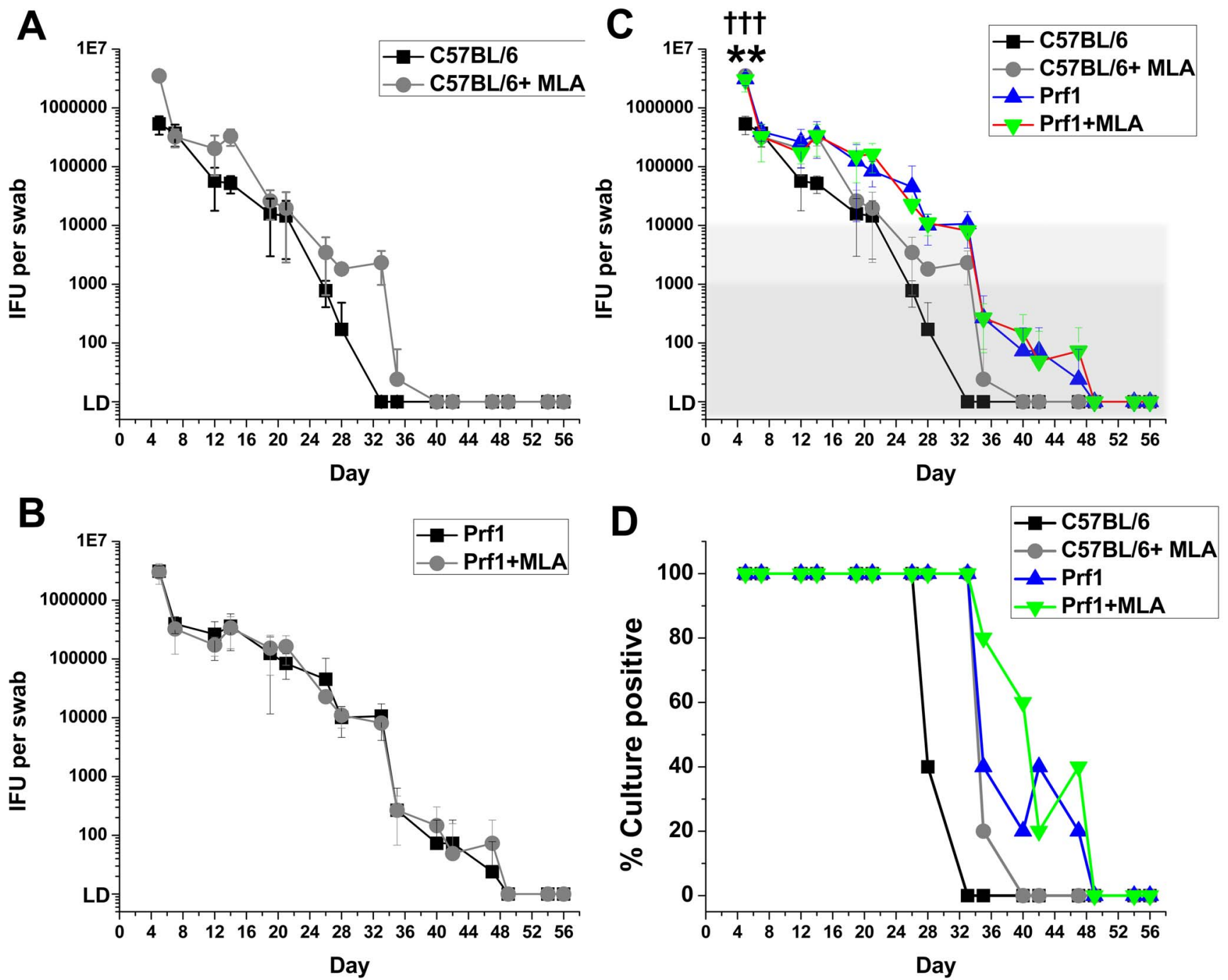

Figure 1. Pharmacologic inhibition of iNOS had no effect on clearance of $C$. muridarum from the genital tract of perforin knockout mice. Wild type C57BL/6J (10 mice; 5 untreated, 5 treated with MLA) and perforin knockout (10 mice; 5 untreated, 5 treated with MLA) female mice were treated with medroxyprogesterone, then infected vaginally one week later with $5 \times 10^{4} \mathrm{IFU}$ of $C$. muridarum. For MLA treatment groups the drinking water contained $50 \mu \mathrm{M}$ MLA (iNOS inhibitor) one day before infection through day 56 post-infection. Genital tract shedding was monitored through day 56 post-infection. The 20 mice in the experiment were inoculated on the same day and monitored in parallel for the duration of the experiment. A) Shedding for C57BL/6J mice. B) Shedding for perforin knockout mice. C) Combined data. D) Combined data for \% culture positive mice over 8 weeks. Differences in the clearance kinetics for C57BL/6J versus perforin knockout mice for the last 5 weeks of the experiment (last 4 logs of clearance, highlighted). Differences between the perforin knockout mice, without or with MLA treatment, and untreated wild type mice were statistically significant with an overall $p$ value $<0.0001 .{ }^{* *}=p$ value $<0.005$ comparing wild type and perforin knockout mice at initial data point (day 5 ). $\dagger \dagger \dagger=p$ value $<0.0005$ comparing untreated wild type mice to wild type mice treated with MLA at initial data point (day 5). doi:10.1371/journal.pone.0063340.g001

buffer) and stored at $-80^{\circ} \mathrm{C}$ until C. muridarum titers were determined on McCoy cell monolayers using an anti-Chlamydia LPS antibody (generous gift of Bobbi Van Der Pol and Jim Williams IUSOM) and FITC-labeled goat anti-mouse IgG (Rockland Immunochemicals; Gilbertsville, PA) as previously described [18]. For the DIC microscopy, arbitrarily selected polyclonal T cell populations, \#5 (wild type) and \#15 (perforindeficient), frozen at the third passage were thawed/expanded then used under identical conditions as the Chlamydia replication/ titration experiments except that instead of harvesting the monolayers at $32 \mathrm{~h}$ post infection the wells were fixed with $10 \%$ formalin and photographed using a Nikon Diaphot 200 inverted microscope equipped with a Diagnostic Instruments spot color camera using the plan20/0.4 DIC lens.

\section{Statistical Analysis}

Summary figures for each experimental investigation are presented means and standard deviations (SD). Figure legends indicate the number of mice or independent $\mathrm{T}$ cell populations per data point in the experiment. Student's two-tailed t-tests and Wilcoxon non-parametric tests, depending on data distribution, were used to assess significance of experimental data. Homogeneity of variances was assessed using a folded F-test. Statistical analysis of Chlamydia shedding was performed using repeated measures analysis of variance to test for differences between groups over time. All data were verified to meet analytic assumptions. Analyses were performed using SAS 9.3 (SAS Institute, Cary, NG). 


\section{Results}

Perforin is not Likely Required for the Plac8-dependent Clearance Mechanism

We tested the role of perforin in Plac 8 -dependent clearance by treating perforin knockout mice with the $i N O S$ inhibitor MLA and infecting them vaginally with $C$. muridarum. In our previous study Plac 8 knockout mice treated with MLA were effectively unable to clear a C. muridarum genital tract infection over 8 weeks, with 11 of 12 mice continuing to shed C. muridarum with an average intensity of $1300 \mathrm{IFU} / \mathrm{swab}$; all wild type C57BL/6 mice treated with MLA, 11 of 11 , cleared the infection by day 40. If the Plac8dependent mechanism were perforin-dependent then perforin knockout mice treated with MLA would have a similarly compromised ability to clear C. muridarum from the genital tract.

$\mathrm{C} 57 \mathrm{BL} / 6 \mathrm{~J}\left(\mathrm{H}-2^{\mathrm{b}}\right)$ and perforin knockout mice $\left(\mathrm{H}-2^{\mathrm{b}}\right)$ were treated with medroxyprogesterone to synchronize estrous, then infected vaginally with $5 \times 10^{4}$ IFU of $C$. muridarum, without or with addition of $i N O S$ inhibitor MLA to the drinking water. Mice were swabbed twice weekly through 56 days, then assessed for genital tract pathology. Recovered IFUs from genital swabs were titered on McCoy monolayers (Fig. 1). Three of five wild type mice cleared the infection by day 28; the remaining two mice by day 33 (next sample collection). Addition of MLA to the drinking water of wild type mice lead to delay in clearance to day 40 , the same day as our previous study. Perforin knockout mice cleared infections on Day 49, and neither the intensity of shedding nor time to clearance were affected by MLA treatment. Wild type and perforin knockout mice, under either treatment condition, were culture negative during the $8^{\text {th }}$ week of the experiment. Perforin knockout mice without or with MLA treatment had higher IFU shedding on initial measurement (day 5) and delayed clearance of C. muridarum from the genital tract compared to untreated wild type mice. At the end of experiment all mice were sacrificed and histopathology was scored. As seen in our previous study, treating wild type C57BL/6 mice with MLA did not change the severity of reproductive tract pathology (data not shown). Perforin knockout mice trended toward less pathology than wild type mice (50\% less; $p$ value $=0.17)$ consistent with previously published data $[8,15]$.

\section{Delayed Clearance in the Perforin Knockout Mice is not Due to $T$ cell Polarization}

Spleens were harvested from all 20 mice on Day 56 to determine whether there was any difference in cytokine polarization related to the absence of perforin or treatment with MLA. Splenocytes were mock-activated (SPG buffer) and activated in vitro with UV-inactivated C. muridarum (stock in SPG buffer); culture supernatants collected at $72 \mathrm{~h}$ for cytokine analysis (Fig. 2). Protective immunity in experimental Chlamydia vaccine studies has previously been shown to been dependent on Thl T cells [19] making IFN- $\gamma$ and TNF- $\alpha$ [20]. IL-10 SNPs have been associated with susceptibility to Chlamydia-mediated tubal infertility in humans [21]. The Th2 cytokine IL-13 has been found to be detrimental in C. muridarum pulmonary infections [22]. There was no significant difference in IFN- $\gamma, \mathrm{TNF}-\alpha$, IL-10, or IL-13 between any of the mice or treatment conditions. MLA had no effect on cytokine polarization. Cytokine polarization does not explain the delayed clearance in perforin knockout mice.

\section{Perforin is Detrimental to the iNOS-dependent Clearance Mechanism in vitro}

Immune $\mathrm{T}$ cells from the 5 untreated wild type and 5 untreated perforin knockout mice in the experiment shown in figure 1 ,

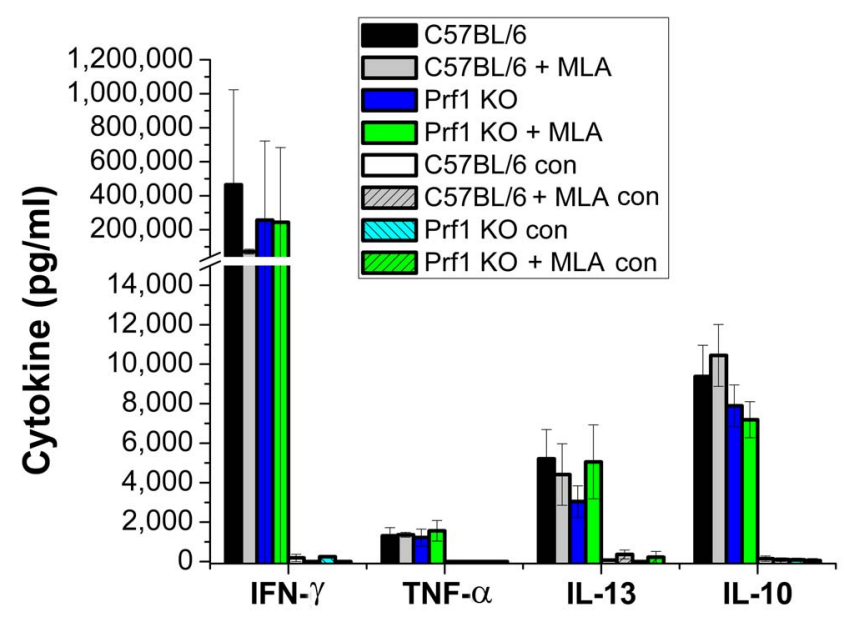

Figure 2. Cytokine profile of immune splenocytes from each experimental group in figure 1 (20 independent splenocyte populations) activated with UV-inactivated C. muridarum. $3 \times 10^{6}$ splenocytes were activated with $7.5 \times 10^{6} \mathrm{UV}$-inactivated C. muridarum (multiplicity of 2.5 IFU per splenocyte) or mock-activated with equivalent volume of SPG buffer $(\mathrm{con}=$ control) in triplicate wells. Supernatants were collected at $72 \mathrm{~h}$ and analyzed by ELISA. Data representing each mouse strain/treatment condition were aggregated and compared to the aggregated data for the untreated C57BL/6J mice. Comparisons were made for each cytokine. There were no statistically significant differences in cytokine polarization between wild type and perforin knockout mice with or without MLA treatment. doi:10.1371/journal.pone.0063340.g002

harvested on day 56, were separately expanded in vitro for 2 passages using UV-inactivated $C$. muridarum and supporting cytokines (see materials and methods). The resulting Chlamydia-specific polyclonal T cell populations were 99\% CD4 T cells (Fig. 3). We tested the ability of these polyclonal populations to terminate $C$. muridarum replication in oviduct epithelial cell line C57epi.1, untreated or pretreated with IFN- $\gamma$, in the absence or presence of the $i N O S$ inhibitor MLA. Epithelial cells were infected with 3 IFU per cell; $4 \mathrm{~h}$ later polyclonal $\mathrm{T}$ cells were added to the wells at an effector to target ratio of 1.25 to $1(250 \mathrm{k} \mathrm{T}$ cells to $\sim 200 \mathrm{~K}$ epithelial cells). $32 \mathrm{~h}$ post infection supernatants were collected, and the wells harvested by scraping in SPG buffer to determine the extent of $C$. muridarum replication; IFU recovered were quantified on McCoy monolayers (Fig. 4). The amount of IFN- $\gamma$ produced by $\mathrm{T}$ cells under each experimental condition was determined by ELISA (Fig. 5). Though not quantifiable, examination of the wells under phase contrast microscopy showed an obvious relative preservation of the epithelial cell monolayer at $32 \mathrm{~h}$ post infection in the wells containing perforin-deficient $\mathrm{T}$ cells compared to wild type T cell wells (Fig. 6), consistent with absence of the perforin the principal effector molecule for $\mathrm{T}$ cell-mediated cytolysis. Because there were more visible inclusions but fewer recovered IFU from the wells containing perforin-knockout $\mathrm{T}$ cells, it is reasonable to suppose that many EB within those visible inclusions were dead. As seen in our previous study [16], overnight treatment of the C57epi.1 epithelial monolayer prior to infection reduced replication by $\sim 4$-fold. In untreated monolayers, perforin-deficient CD4 $\mathrm{T}$ cells were 5 times more efficient at terminating C. muridarum replication in epithelial cells than were wild type $\mathrm{T}$ cells. That difference in efficiency was blunted somewhat by pretreating the epithelial cell monolayers with IFN- $\gamma$, boosting epithelial nitric oxide production and MHC class II expression [17]. Both wild type and perforin-deficient early passage polyclonal $\mathrm{T}$ cell populations used the iNOS-dependent mechanism to terminate 
replication as addition of MLA to the experimental wells essentially abrogated their ability to terminate Chlamydia replication. In the presence of $1 \mathrm{mM}$ MLA both polyclonal $\mathrm{T}$ cell populations reduced recovered IFU by only 2 -fold. This result suggests that CD4 T cells capable of $i$ NOS-independent termination of replication were present in numbers below that necessary to efficiently control replication in the presence of MLA. Consistent with, or in addition to, their greater efficiency in terminating replication, perforin-deficient $\mathrm{T}$ cells made $\sim 2$-fold more IFN- $\gamma$ under all experimental conditions (see Fig. 5).

\section{Discussion}

Studies of protective immunity against Chlamydia infections of the genital tract have been confounded by the existence of three independent and singly sufficient mechanisms for clearing $C$. muridarum (reviewed in [23]). The first mechanism discovered is dependent on T cell- epithelial cell contact and IFN- $\gamma-$ mediated up regulation of epithelial nitric oxide production to chlamydiacidal levels [10]. The second mechanism described is $\mathrm{T}$ cellindependent once established [24]; it is dependent on Chlamydiaspecific antibodies [25] and likely Fc receptors [26]. The third mechanism discovered is dependent on Plac 8 [13] and likely CD4 $\mathrm{T}$ cell degranulation [16].

Based on the previous work of others showing no difference in clearance between C57BL/6 and perforin knockout mice $[8,15]$, and our work showing a dramatically diminished ability to clear $C$. muridarum in Placo knockout mice treated with MLA [13], we sought to definitively test whether the Plac8-dependent mechanism was dependent on perforin by comparing clearance in perforin knockout mice without and with $i N O S$ inhibitor treatment. Our experiments show that perforin knockout mice had delayed clearance compared to wild type mice, and that MLA treatment had no effect on clearance in the perforin knockout mice. Delayed clearance in perforin knockout mice could not be attributed to a difference in cytokine polarization and was not associated with greater immunopathology.

The finding of delayed clearance in perforin knockout mice differs between Perry et al [15] and our current study, likely reflecting the inoculating dose; 1500 IFU per mouse and 50,000 IFU per mouse respectively. In support of that possibility, Perry et al saw no difference in wild type and perforin knockout mice genital tract IFU at their initial time point; in our study there was 6 -fold higher IFU in the perforin knockout mice than wild type mice on day 5. Our results also differ from those of Murthy et al. [8] who used an inoculating dose of 50,000 IFU, but treated mice with medroxyprogesterone on day T-10 and day T-3 prior to infection. In their model, IFU recovered on day five are $\sim 20,000 /$ swab while our recovered IFU on day 5 are $\sim 2,000,000 /$ swab. It is likely that the higher effective inoculating dose in our experimental model (based on inoculum size or hormonal manipulation) revealed defects in clearance not seen in the two other models.

The data in figure 4 show that perforin is detrimental to the iNOS-dependent mechanism for terminating Chlamydia replication in epithelial cells, either because cytolysis of the epithelial target decreases $\mathrm{T}$ cell activation and IFN- $\gamma$ production, or more likely, inserting perforin channels (punching holes) in the epithelial membrane lowers the effective concentration or duration of exposure of Chlamydia to epithelial cell-produced intracellular nitric oxide. 5-fold more efficient replication termination by perforindeficient $\mathrm{T}$ cells occurred in the setting of diminished cytolysis (see Fig. 6). More intact epithelial cells with visible inclusions were seen in perforin-deficient $\mathrm{T}$ cell wells, but fewer viable IFU were recovered from them suggesting that $\mathrm{EB}$ within those inclusions were dead; a counter intuitive result for conventional intracellular pathogen host defense paradigms. Because the IFN- $\gamma$ levels were low $(1-2 \eta \mathrm{g} / \mathrm{ml})$, and only increased $\sim 2$-fold in perforin-deficient experimental wells, we postulate that the 5 -fold greater potency of perforin-deficient $\mathrm{T}$ cells reflects a detrimental effect of perforin on functional levels of microbicide within infected cells. In support of that postulate, we have previously shown that addition of IFN- $\gamma$ to our reproductive tract epithelial cell lines 4 or more hours after infection had no effect on $C$. muridarum replication or on the replication of the much more IFN- $\gamma$ sensitive human $C$. trachomatis serovars D and L2 [27]. In these current experiments we added the $\mathrm{T}$ cells to the infected monolayers $4 \mathrm{~h}$ post-infection. We have also previously shown in 4 of 4 Chlamydia-specific $\mathrm{T}$ cell clones that the earliest recognition of untreated infected epithelial cells as measured by IFN- $\gamma$ production is between $15-18 \mathrm{~h}$ post-infection $[17,28]$. In our current experiment, IFN- $\gamma$ in meaningful levels were likely non-existent until $\sim 20 \mathrm{~h}$ into the experiment, a time point at which the large majority of $C$. muridarum replication is already complete in C57epi.1 cells [17]. As a working model we propose that CD4 $\mathrm{T}$ cell-mediated termination of Chlamydiareplication in epithelial cells occurs via a toxic bag type mechanism. T cells form conjugates with infected epithelial cells, activating targeted cells to make nitric oxide; punching holes in the plasma membrane and/or lysing the epithelial cell is detrimental to this mechanism (Fig. 7).

Perforin-deficient mice treated with MLA did not behave like Plac8-deficient mice treated with MLA. Our previous experience was that $P l a c 8$ knockout mice treated with MLA could not clear $C$. muridarum within 8 weeks, with substantial levels (1300 IFU/swab) of ongoing IFU shedding at the end of week 8, day 56. All perforin-deficient mice, untreated or treated with MLA, cleared their infection by day 49 with no measurable IFU shedding during the $8^{\text {th }}$ week of the experiment. Because untreated perforin knockout mice unexpectedly differed from C57BL/6 mice, we could not performed the planned statistical analysis comparing perforin-deficient mice to perforin-deficient mice treated with
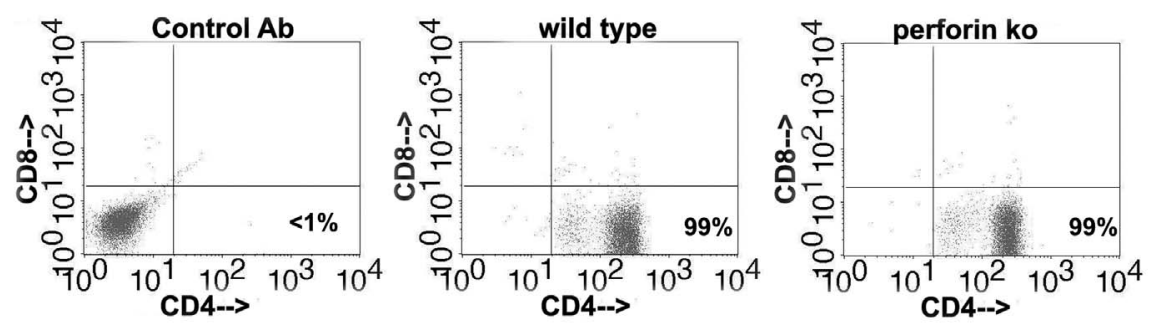

Figure 3. Chlamydia-specific polyclonal T cell populations derived from immune mice are $99 \% \mathrm{CD4}^{+} \mathrm{CD8}^{-}$after three passages with UV-inactivated $C$. muridarum and supporting cytokines regardless of perforin status. doi:10.1371/journal.pone.0063340.g003 

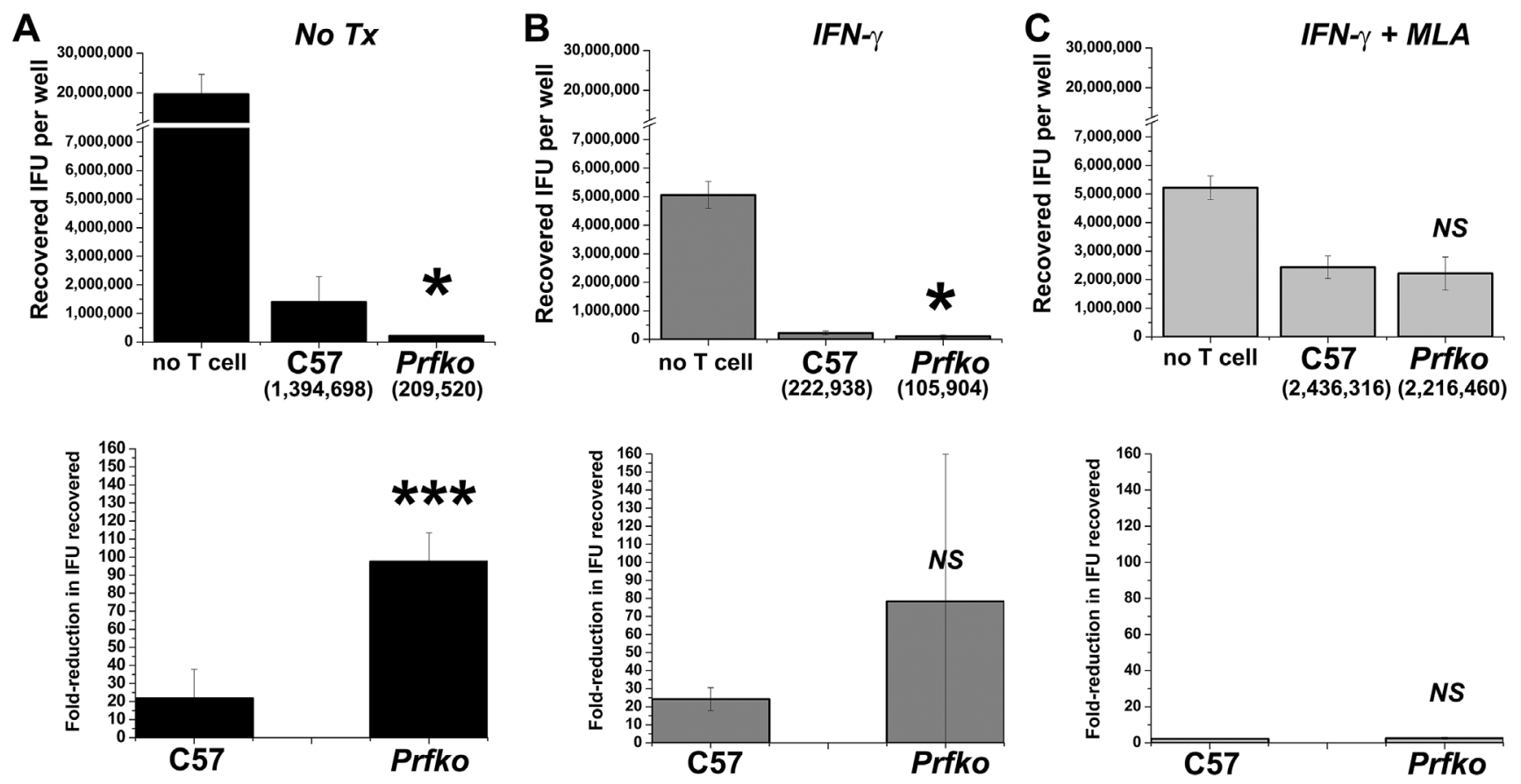

Figure 4. Perforin-deficient CD4 T cells are superior to wild type CD4 T cells for terminating C. muridarum replication in epithelial cells. Top panels show IFU recovered per well, with means for the experimental wells shown in parentheses below their respective bars. The bottom panels show the T cell-mediated fold-reduction in IFU (calculated as IFU recovered from the "no T cell" control wells/IFU recovered in the experimental wells). A) Untreated C57epi.1 monolayers. B) C57epi.1 monolayers treated with $10 \eta \mathrm{g}$ recombinant murine IFN- $\gamma$ for $10 \mathrm{~h}$ prior to infection. C) C57epi.1 monolayers treated with $10 \mathrm{\eta g}$ recobinant murine IFN- $\gamma$ and $1 \mathrm{mM} \mathrm{MLA}$ for $10 \mathrm{~h}$ prior to infection. All monolayers were infected with C. muridarum at 3 IFU per cell. Inocula were removed $4 \mathrm{~h}$ later and infected epithelial cells were co-cultured without (no T cells) and with $2.5 \times 10^{5} \mathrm{~T}$ cells in the absence and presence of MLA as indictated. $32 \mathrm{~h}$ post infection supernatants were collected and the wells harvested; $C$. muridarum was quantified on McCoy monolayers. In a single experiment, each experimental condition (no treatment, IFN- $\gamma$ pretreatment, IFN- $\gamma$-MLA pretreatment) was done as triplicates for each of the $10 \mathrm{~T}$ cell lines and the "no T cell" control. Comparisons were made between aggregated data for the 5 wild type T cell lines (C57) and the 5 perforin knockout T cell lines (PrfKo) for each experimental condition. ${ }^{*}=p$ value $<0.05$; ${ }^{* * *}=p$ value $<0.0005 ; N S=$ not statistically significant.

doi:10.1371/journal.pone.0063340.g004

MLA to assess whether perforin was critical to the Plac8-dependent mechanism. However, it is clear from the data here that there was no difference in clearance between a perforin knockout mouse and

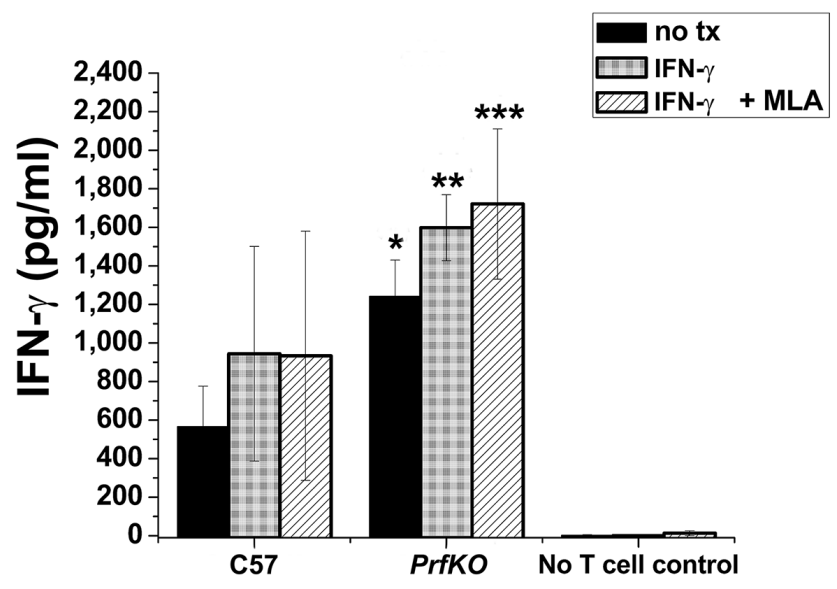

Figure 5. IFN- $\gamma$ levels for each treatment condition in fi $\gamma$ ure 4 as determined by ELISA. MLA treatment had no statistically significant effect on wild type or perforin-deficient $T$ cells compared to the untreated control for each T cell type. * are for comparisons to ' $C 57$ no tx' for all conditions; ${ }^{*}=p$ value $<0.05 ;{ }^{* *}=p$ value $<0.005 ;{ }^{* *}=p$ value $<0.0005 ; N S=$ not statistically significant.

doi:10.1371/journal.pone.0063340.g005 a perforin knockout mouse treated with MLA. Had the Plac8dependent mechanism been dependent on perforin, we would have expected MLA-treated perforin knockout mice to shed Chlamydia throughout the $8^{\text {th }}$ week of the experiment; they did not. The delayed clearance in untreated or MLA-treated perforin knockout mice compared to wild type mice is unlikely to represent a compromised Placo-dependent clearance mechanism that is strongly associated with the in vitro degranulation-dependent termination mechanism $[13,16]$. Perforin's contribution to bacterial clearance is not likely occurring through enhancing CD4 T cell termination of Chlamydia replication in epithelial cells as it does not appear to relevant to the Plac8-dependent mechanism and is detrimental to the $i N O S$-dependent mechanism.

$\mathrm{T}$ cells, mast cells, eosinophils, basophils, CD4negative NKT cells and NK cells express perforin. NK cells recognize and lyse infected epithelial cells [29,30], but NK cells by themselves have only a modest ability to terminate replication in epithelial cells in vitro ( $\sim 2$-fold) [30]. However, cytolysis of infected cells by any means would expose intracellular Chlamydia EB to extracellular defense mechanisms including complement. Chlamydia trachomatis EB activate the alternative complement pathway and C3b covalently binds to MOMP [31], and early components of complement upstream of the membrane attack complex neutralize Chlamydia EB in vitro [32]. While existing data argue against complement being singly sufficient for clearing genital tract infections, as $\mathrm{T}$ cell deficient mice sufficient in complement cannot clear infections, complement could logically contribute to 

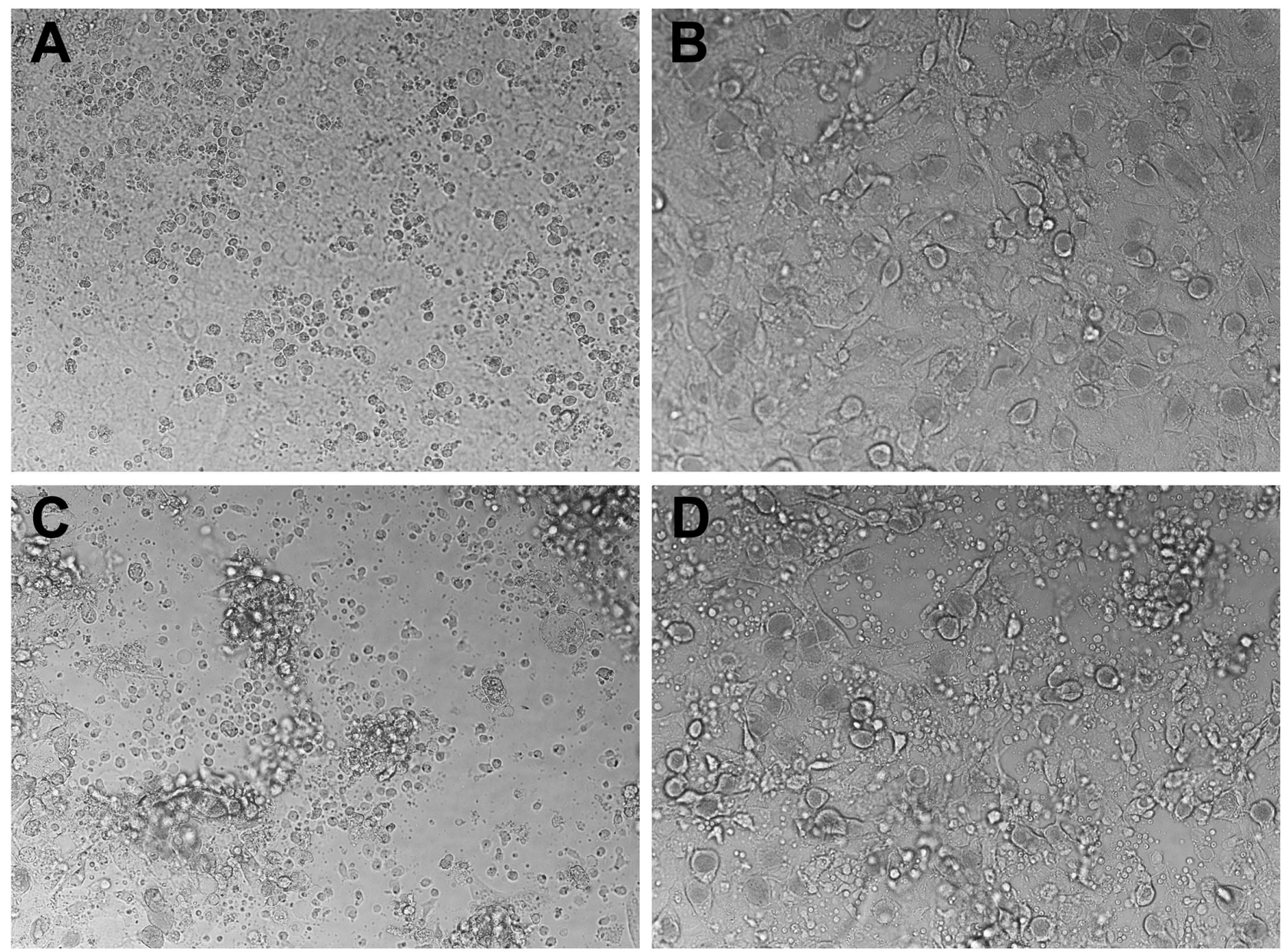

Figure 6. Perforin-deficient CD4 T cells have a lesser cytopathic effect on infected C57 epithelial monolayers. A) Uninfected C57epi.1 monolayer at $32 \mathrm{~h}$ post mock infection (overgrown confluent monolayer). B) C57epi. 1 monolayer $32 \mathrm{~h}$ post infection with C. muridarum at $3 \mathrm{IFU}$ per cell. C) Infected C57epi.1 monolayer co-cultured with wild type polyclonal CD4 T cells at an effector to target ration of 1.25:1 at $32 \mathrm{~h}$ post infection. D) Infected C57epi.1 monolayer co-cultured with perforin-deficient polyclonal CD4 T cells at an effector to target ration of 1.25:1 at 32 h post infection. Representative DIC images at 200x magnification.

doi:10.1371/journal.pone.0063340.g006

\section{Wild type}

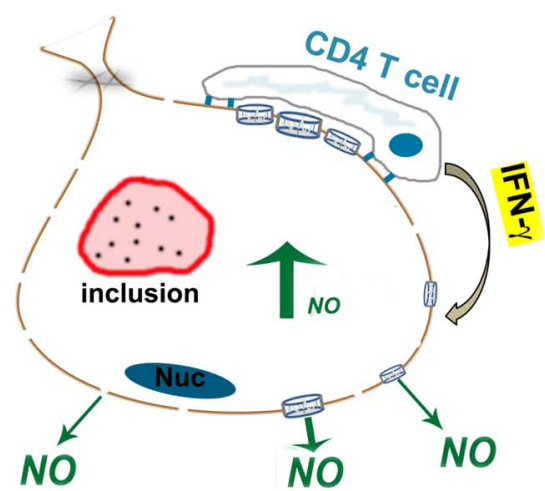

\section{Perforin KO}

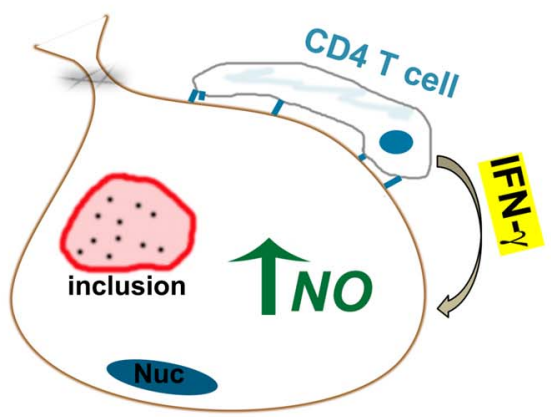

Figure 7. The toxic bag paradigm for CD4 T cell termination of replication in epithelial cells. Chlamydia-specific wild type T cells damage epithelial membrane integrity by inserting perforin channels and activating apoptotic pathways within target cells. Effectiveness of nitric oxide termination of replication is concentration dependent. Perforin-mediated damage to the membrane likely lowers the effective concentration of nitric oxide within the target cell, diminishing the relative potency of wild type CD4 T cells compared to perforin-deficient CD4 T cells. Nuc = nucleus. doi:10.1371/journal.pone.0063340.g007 
substantial reductions in viable extracellular EB in genital tract secretions. A recent study in a murine $C$. psittaci model showing a major contribution of C3 to protective immunity [33] raises the question of whether previous unpublished negative data based on cobra venom depletion of the complement membrane attack complex referred to in Williams et al [34] sufficiently addressed the role of early complement components in innate Chlamydia immunity.

\section{Conclusion}

During experimental C. muridarum genital tract infections perforin plays a complex role. Others have shown that it

\section{References}

1. (2011) Sexually Transmitted Disease Surveillance 2010. In: Prevention CfDCa, editor. Atlanta, GA: U.S. Department of Health and Human Services.

2. Brunham RC, Pourbohloul B, Mak S, White R, Rekart ML (2005) The Unexpected Impact of a Chlamydia trachomatis Infection Control Program on Susceptibility to Reinfection. J Infect Dis 192: 1836-1844.

3. Brunham RC, Rekart ML (2008) The arrested immunity hypothesis and the epidemiology of chlamydia control. Sex Transm Dis 35: 53-54.

4. Rekart ML, Brunham RC (2008) Epidemiology of chlamydial infection: are we losing ground? Sex Transm Infect 84: 87-91.

5. Schachter J (1985) Overview of Chlamydia trachomatis infection and the requirements for a vaccine. Rev Infect Dis 7: 713-716.

6. Morrison RP, Caldwell HD (2002) Immunity to murine chlamydial genital infection. Infect Immun 70: 2741-2751.

7. Igietseme JU, He Q Joseph K, Eko FO, Lyn D, et al. (2009) Role of T lymphocytes in the pathogenesis of Chlamydia disease. J Infect Dis 200: 926934.

8. Murthy AK, Li W, Chaganty BK, Kamalakaran S, Guentzel MN, et al. (2011) Tumor necrosis factor alpha production from CD8+ T cells mediates oviduct pathological sequelae following primary genital Chlamydia muridarum infection. Infect Immun 79: 2928-2935.

9. Igietseme JU (1996) The molecular mechanism of T-cell control of Chlamydia in mice: role of nitric oxide. Immunology 87: 1-8.

10. Igietseme JU, Uriri IM, Hawkins R, Rank RG (1996) Integrin-mediated epithelial-T cell interaction enhances nitric oxide production and increased intracellular inhibition of Chlamydia. J Leukoc Biol 59: 656-662.

11. Igietseme JU, Perry LL, Ananaba GA, Uriri IM, Ojior OO, et al. (1998) Chlamydial infection in inducible nitric oxide synthase knockout mice. Infect Immun 66: 1282-1286.

12. Ramsey KH, Miranpuri GS, Poulsen GE, Marthakis NB, Braune LM, et al. (1998) Inducible nitric oxide synthase does not affect resolution of murine chlamydial genital tract infections or eradication of chlamydiae in primary murine cell culture. Infect Immun 66: 835-838.

13. Johnson RM, Kerr MS, Slaven JE (2012) Plac8-dependent and inducible NO synthase-dependent mechanisms clear Chlamydia muridarum infections from the genital tract. J Immunol 188: 1896-1904.

14. Ramsey KH, Miranpuri GS, Sigar IM, Ouellette S, Byrne GI (2001) Chlamydia trachomatis persistence in the female mouse genital tract: inducible nitric oxide synthase and infection outcome. Infect Immun 69: 5131-5137.

15. Perry LL, Feilzer K, Hughes S, Caldwell HD (1999) Clearance of Chlamydia trachomatis from the murine genital mucosa does not require perforin-mediated cytolysis or Fas-mediated apoptosis. Infect Immun 67: 1379-1385.

16. Jayarapu K, Kerr M, Ofner S, Johnson RM (2010) Chlamydia-specific CD4 T cell clones control Chlamydia muridarum replication in epithelial cells by nitric oxide-dependent and -independent mechanisms. J Immunol 185: 6911-6920.

17. Jayarapu K, Kerr MS, Katschke A, Johnson RM (2009) Chlamydia muridarumspecific CD4 T-cell clones recognize infected reproductive tract epithelial cells in an interferon-dependent fashion. Infect Immun 77: 4469-4479.

18. Johnson RM (2004) Murine oviduct epithelial cell cytokine responses to Chlamydia muridarum infection include interleukin-12-p70 secretion. Infect Immun 72: 3951-3960. contributes to the immunopathogenesis of scarring [8]. Here we show that perforin contributes to bacterial clearance, likely by a mechanism that does not involve CD4 $\mathrm{T}$ cell interactions with infected epithelial cells.

\section{Author Contributions}

Conceived and designed the experiments: RMJ JES. Performed the experiments: RMJ MSK. Analyzed the data: JES. Wrote the paper: RMJ. Manuscript editing and revision: RMJ MSK JES.

19. Hawkins RA, Rank RG, Kelly KA (2002) A Chlamydia trachomatis-specific Th2 clone does not provide protection against a genital infection and displays reduced trafficking to the infected genital mucosa. Infect Immun 70: 5132-5139.

20. Yu H, Karunakaran KP, Kelly I, Shen C, Jiang X, et al. (2011) Immunization with live and dead Chlamydia muridarum induces different levels of protective immunity in a murine genital tract model: correlation with MHC class II peptide presentation and multifunctional Th1 cells. J Immunol 186: 3615-3621.

21. Kinnunen AH, Surcel HM, Lehtinen M, Karhukorpi J, Tiitinen A, et al. (2002) HLA DQ alleles and interleukin-10 polymorphism associated with Chlamydia trachomatis-related tubal factor infertility: a case-control study. Hum Reprod 17: 2073-2078.

22. Asquith KL, Horvat JC, Kaiko GE, Carey AJ, Beagley KW, et al. (2011) Interleukin-13 promotes susceptibility to chlamydial infection of the respiratory and genital tracts. PLoS Pathog 7: e1001339.

23. Johnson RM (2012) Chlamydial Infections. In: Eugene Rosenberg EFD, Fabiano Thompson, Stephen Lory, Erko Stackebrandt editor. The Prokaryotes, 4th Edition. NY: Springer Publishing Co.

24. Morrison SG, Morrison RP (2001) Resolution of secondary Chlamydia trachomatis genital tract infection in immune mice with depletion of both CD4+ and CD8+ T cells. Infect Immun 69: 2643-2649.

25. Morrison SG, Morrison RP (2005) A predominant role for antibody in acquired immunity to chlamydial genital tract reinfection. J Immunol 175: 7536-7542.

26. Moore T, Ananaba GA, Bolier J, Bowers S, Belay T, et al. (2002) Fc receptor regulation of protective immunity against Chlamydia trachomatis. Immunology 105: 213-221.

27. Nelson DE, Virok DP, Wood H, Roshick C, Johnson RM, et al. (2005) Chlamydial IFN-\{gamma\} immune evasion is linked to host infection tropism. Proc Natl Acad Sci U S A 102: 10658-10663.

28. Johnson RM, Yu H, Kerr MS, Slaven JE, Karunakaran KP, et al. (2012) PmpG303-311, a protective vaccine epitope that elicits persistent cellular immune responses in Chlamydia muridarum-immune mice. Infect Immun 80: 2204-2211.

29. Hook CE, Telyatnikova N, Goodall JC, Braud VM, Carmichael AJ, et al. (2004) Effects of Chlamydia trachomatis infection on the expression of natural killer (NK) cell ligands and susceptibility to NK cell lysis. Clin Exp Immunol 138: 54 60.

30. Ibana JA, Aiyar A, Quayle AJ, Schust DJ (2012) Modulation of MICA on the surface of Chlamydia trachomatis-infected endocervical epithelial cells promotes NK cell-mediated killing. FEMS Immunol Med Microbiol 65: 32-42.

31. Hall RT, Strugnell T, Wu X, Devine DV, Stiver HG (1993) Characterization of kinetics and target proteins for binding of human complement component C3 to the surface-exposed outer membrane of Chlamydia trachomatis serovar L2. Infect Immun 61: 1829-1834

32. Lin JS, Yan LL, Ho Y, Rice PA (1992) Early complement components enhance neutralization of Chlamydia trachomatis infectivity by human sera. Infect Immun 60: 2547-2550.

33. Bode J, Dutow P, Sommer K, Janik K, Glage S, et al. (2012) A New Role of the Complement System: C3 Provides Protection in a Mouse Model of Lung Infection with Intracellular Chlamydia psittaci. PLoS ONE 7: e50327.

34. Williams DM, Schachter J, Weiner MH, Grubbs B (1984) Antibody in host defense against mouse pneumonitis agent (murine Chlamydia trachomatis). Infect Immun 45: 674-678. 